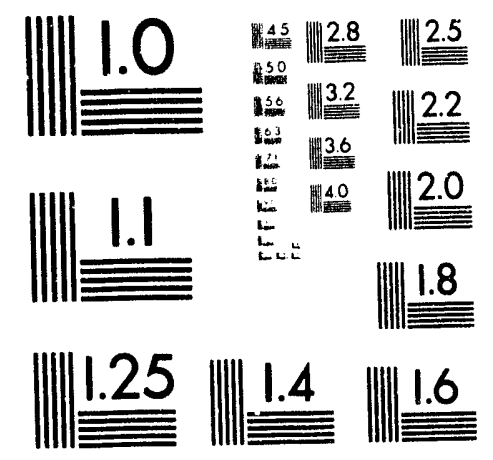



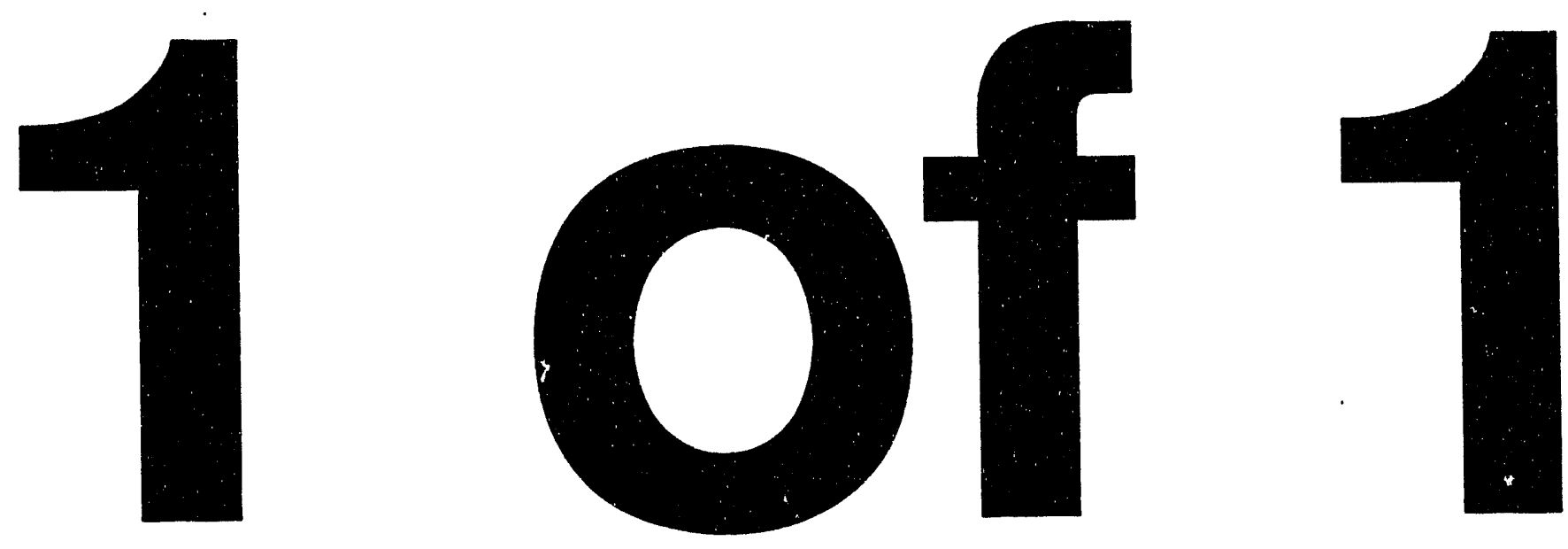


\section{SEP 22 W5}

OSTI

\section{INSTITUTE FOR FUSION STUDIES}

DOE/ET-53088-619

Effect of Limiter End Loss in Finite

Larmor Radius Theory

H.L. BERK

Institute for Fusion Studies

The University of Texas at Austin

Austin, Texas 78712 and

I.A. Kotelnikov

Budker Institute of Nuclear Physics

630090, Novosibirsk, Russia

\section{THE UNIVERSITY OF TEXAS}

\section{AUSTIN}

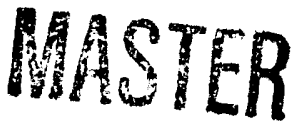




\title{
Effect of Limiter End Loss in Finite Larmor Radius Theory
}

\author{
H.L. Berk \\ Institute for Fusion Studies \\ The University of Texas at Austin \\ Austin, Texas 78712 \\ and \\ I.A. Kotelnikov \\ Budker Institute of Nuclear Physics \\ 630090, Novosibirsk, Russia
}

\begin{abstract}
We have examined the effect of incomplete line tying on the MHD flute mode with FLR (finite Larmor radius) effects. We show that the combination of line tying and FLR effects can slow down MHD instability, but cannot produce complete stabilization.

\section{DISCLAIMER}

This repert was prepared as an account of work sponsored by an agency of the United States Government. Neither the United States Government nor any agency thereof, nor any of their employees, makes any warranty, express or implied, or assumes any legal liability or responsibility for the accuracy, completeness, or usefulness of any information, apparatus, product, or process disclosed, or represents that its use would not infringe privately owned rights. Reference herein to any specific commercial product, process, or service by trade name, trademark, manufacturer, or otherwise does not necessarily constitute or imply its endorsement, recommanufacturer, or favoring by the United Status Government or any agency thereof. The views and opinions of authors expressed herein do not necessarily state or reflect those of the United States Government or any agency thereof.
\end{abstract}


It is well known that finite Larmor radius (FLR) consideration can strongly modify the predictions of MHD theory. ${ }^{1,2}$ However, even when FLR effects dominate the response of a plasma confined system, the finite Larmor radius effects by themselves cannot stabilize the $m=1$ displacement mode. At relatively high beta, stabilization can be achieved by taking into account the image current from conducting walls ${ }^{3,4,5}$ without direct contact with the walls. Even in this case a slow instability persists due to the finite resistive decay time of the conducting wall. ${ }^{6}$ Alternatively, the plasma can be in direct contact with a radial external wall. ${ }^{7} 8$ The plasma is then unable to move at the edge. Thus, as the overall displacement cannot be rigid, some direct FLR effects will persist even for the $m=1$ mode, which makes stabilization in principle possible.

In this work we analyze the effect of an edge limiter on FLR stabilization. It will be assumed that the hot species of the plasma is confined in a mirror machine, with the axial turning points inside the limiters (Fig. 1), so that the confinement of the hot particles is not directly affected by the limiter. In addition the hot particles are immersed in a bath of background plasma. This background plasma is being scraped-off by the axial limiters. We will describe the axial loss according to the theory of the Kunkel-Guillory sheath, ${ }^{10}$ which causes a surface current in response to plasma perturbations. If this surface current is large enough, the field lines intersecting the limiter will be shorted, and the plasma will be unable to move at the edge as the $\mathbf{E} \times \mathbf{B}$ drift is zero there. Then the overall plasma response is the same as described in Refs. 7 and 8. However, in general the shorting is not complete, and some plasma motion at the edge can occur. We will see that incomplete end shorting causes the persistence of instability, though at a level considerably reduced from the case where no limiters are present.

We consider the plasma to consist of a core component (we use the subscript " $c$ " to refer to this component) and a hot particle component (we use the subscript " $h^{n}$ for this 
component). Typically the hot particle density, $n_{k}$, is mach less than the core electron density, $n_{e}$. We assume $1 \ll\left(\partial \beta_{h} / \partial r\right) / \kappa, 1 \ll\left(\partial \beta_{c} / \partial r\right) / \kappa$, with $\beta_{j}$ the beta value of the component $j$ and $\kappa$ is the field line curvature.

To describe the response of FLR and other terms we start from a variational form frequently referred to as the Lagrangian. ${ }^{10-12,5}$ For a symmetric machine satisfying the paraxial approximation, the Lagrangian form in terms of the perturbed eigenfunction $\phi(\psi, s) \exp (-i \omega t+$ imo), takes the form

$$
\begin{aligned}
\mathcal{L} \equiv & 2 \pi \int \frac{d \psi d s}{B}\left\{\frac{\sigma}{4 \pi} \frac{m^{2}}{r^{2}}\left(\frac{\partial \phi}{\partial s}\right)^{2}+\frac{\sigma r^{2} B^{2}}{4 \pi}\left[\frac{\partial}{\partial s}\left(\frac{\partial \phi}{\partial \psi}-\frac{\phi}{B} \frac{\partial B}{\partial \psi}\right)\right]^{2}\right. \\
& -\frac{\kappa m^{2} \phi^{2}}{r B} \frac{D}{D \psi}\left(P_{\perp}+P_{\|}\right) \\
& -\sum_{j} n_{j} m_{j} \omega^{2}\left[\left(r B \frac{\partial}{\partial \psi} \frac{\phi}{B}\right)^{2}+\frac{m^{2} \phi^{2}}{r^{2} B^{2}}\right] \\
& \left.-\sum_{j} n_{j} m_{j} A_{j}\left[r^{4} B^{2}\left(\frac{\partial}{\partial \psi} \frac{\phi}{r B}\right)^{2}+\left(m^{2}-1\right)\left(\frac{\phi}{r B}\right)^{2}\right]\right\} \\
& +\mathcal{L}_{\text {loss }}+\mathcal{O}\left(\kappa^{2} r^{2} / \beta\right) .
\end{aligned}
$$

Her $\mathbf{B}=\nabla \psi \times \nabla \theta, \psi=\int_{0}^{r} d r^{\prime} r^{\prime} B_{z}\left(r^{\prime}, z\right), \theta$ the azimuthal coordinate, $B$ is the magnitude of the magnetic field, $r$ is the radial coordinate, $s$ is the distance along a field line, $\sigma=1+$ $4 \pi\left(P_{\perp}+P_{\|}\right) / B^{2}, \kappa=\nabla \psi \cdot(\mathbf{b} \cdot \nabla) \mathbf{b} /|\nabla \psi|$ is the field line curvature, $\mathbf{b}=\mathbf{B} / B$ unit vector along $\mathbf{B}, P_{\perp}$ and $P_{\|}$are the perpendicular and parallel pressure, $\frac{D}{D \psi}(\quad) \equiv \nabla \psi \cdot \nabla(\quad) /|\nabla \psi|^{2}$, $\phi=\boldsymbol{\xi} \cdot \nabla \psi / m$ with $\boldsymbol{\xi} \equiv i c \mathbf{E} \times \mathbf{b} / \omega B$ the perturbed displacement and $\mathbf{E}$ the perturbed electric field $m$ is the azimuthal mode number, $\omega$ the mode frequency, $n_{j}$ and $m_{j}$ are the density and mass of component $j$, and $A_{j}$ is the FLR term given by

$$
\begin{gathered}
A_{j}=-\frac{m \omega}{n_{j} m_{j} \omega_{c j}} \frac{D}{D \psi}\left(P_{\perp j} B\right)+\frac{m^{2}}{2 n_{j}^{2} m_{j}^{2} \omega_{c j}^{2}} B \frac{\partial B}{\partial \psi} \frac{D L_{j}}{D \psi}, \quad \omega_{c j}=\frac{q_{j} B}{m_{j} c}, \\
P_{\perp j}=m_{j} \int d^{3} v F_{j} v_{\perp}^{2} / 2, \quad \mathcal{L}_{j}=\frac{m_{j}^{2}}{4} \int d^{3} v F_{j} v_{\perp}^{4}
\end{gathered}
$$


with $F_{j}$ the equilibrium distribution of species $j$ and $m_{j}$ the mass of species $j$. For compactness we write $A_{j}$ in the schernatic form

$$
A_{j}=-\omega \omega_{*_{j}}\left(1-\beta_{j} / 2\right)-\overline{\omega_{*_{j}}^{2} \beta_{j}} / 2
$$

where $\omega_{*_{j}}=\frac{m B}{n_{j} m_{j} \omega_{c j}} \frac{D P_{\perp j}}{D \psi}$. If $F_{j}$ is a Maxwellian at constant temperature then $\overline{\omega_{*_{j}}^{2} \beta_{j}} / 2=$ $\omega_{* j}^{2} \beta_{j} / 2$ with $\beta_{j}=8 \pi P_{\perp j} / B^{2}$.

In the Langrangian form given by Eq. (1) we have added a term that takes into account particle end loss. This additional term has been derived in Ref. 13 and it is of form

$$
\mathcal{L}_{\text {loss }}=-\frac{i}{c} \int d S \mathbf{n} \cdot \mathbf{b} \delta j_{\|} \phi
$$

where $S$ is the surface area of the wall material that intersects the magnetic field lines of the plasma, $\mathrm{n}$ is the normal to the surface, $\mathrm{t}=\mathbf{B} / B$, and $\delta j_{\|} \exp (-i \omega t+i m \theta)$ is the perturbed plasma end current density that flows between the plasma and the end-conducting walls. For the Kunkel-Guillory sheath model ${ }^{9}$

$$
\delta j_{\|}=e^{2} n_{e} v_{i} \frac{\epsilon(\psi) \phi \omega}{2 \sqrt{\pi} T_{e} c}
$$

where $e$ is electron charge, $v_{i}=\sqrt{2 T_{i} / m_{i}}, n_{e}$ the electron density, $\epsilon(\psi) \simeq 1$ where the limiter intersects the plasma and $\epsilon(\psi)=0$ on field lines where there is no limiter. Using that $d S=2 \pi d \psi / B$, the loss term in the Lagrangian becomes

$$
\mathcal{L}_{\text {loss }}=-2 \pi i e^{2} \frac{\omega}{c^{2}} \int \frac{d \psi d s}{B} \frac{n_{e} v_{i} \epsilon(\psi)}{2 \sqrt{\pi} T_{e}} \sum_{\text {lim }} \delta\left(s-s_{\text {lim }}\right) \phi^{2}
$$

where the summation is on limiters' positions. The Lagrangian form (1) can be viewed as a global dispersion relation with $\mathcal{L}=0$ and as a rariational expression, where the Euler equation is determined by $\delta \mathcal{L} / \delta \phi=0$.

Let us now estimate the order of magnitude of the Lagrangian terms in Eq. (1) for the $m=1$ mode (we exclude the loss term in this estimate). Taking $\frac{\partial}{\partial s} \sim \frac{1}{L}, \frac{\partial}{\partial \psi} \sim \frac{1}{B r^{2}}$, 
$\kappa \sim \frac{r}{L^{2}}, \delta \sim n_{h} / n_{c}, T_{h} / T_{c} \equiv$ hot to background temperature ratio, $a_{i} \equiv$ Larmor radius of background ions, we then find that the estimated ratio for first four bracketed terms in Eq. (1), i.e. the ratio of the honding energy, to the curvature drive, to the plasma inertia, to the FLR stabilization is respectively

$$
1: \beta: \beta \omega^{2} L^{2} / v_{i}^{2}: \beta L^{2} \omega a_{i}\left(1+\delta T_{h} / T_{c}\right) / r^{2} v_{i}+\beta^{2} L^{2} a_{i}^{2}\left(1+\delta T_{h}^{2} / T_{c}^{2}\right) / r^{4}
$$

To implement a self-consistent analysis we cake an ordering where

$$
\beta^{2} \ll \frac{r^{4}}{L^{2} a_{i}^{2}\left(1+\delta T_{h}^{2} / T_{c}^{2}\right)} \ll \beta \ll \frac{r^{2}}{L a_{i}\left(1+\delta T_{h}^{2} / T_{c}^{2}\right)^{1 / 2}} \ll 1 .
$$

Note that in this ordering the beta dependent FLR term is comparable or larger than the frequency dependent FLR term. Observe that the bending energy dominates the Lagrangian. This term is annihilated to an insignificant order $\left(\mathcal{O}\left(\beta^{2}\right)\right)$ by choosing $\phi(\psi, s)$ to be independent of $s$.

The next largest term is the one due to FLR, which is annihilated by choosing to leading o. $\operatorname{ter} \phi(\psi)=r B / \sqrt{2}=B_{0}^{1 / 2}(s) \psi^{1 / 2}+\mathcal{O}(\beta)$. However, in tilis order we will also take into account the changes in $\phi$ due to other terms in the Lagrangian. Hence, to obtain the appropriate Euler equation we take the variation with respect to $\phi$, with the constraint that it is independent of $s$, and find

$$
\begin{gathered}
\frac{2}{\psi^{1 / 2}} \frac{\partial}{\partial \psi} \overline{\mathcal{N} A} \psi^{2} \frac{\partial}{\partial \psi}\left(\frac{\phi}{\psi^{1 / 2}}\right)+2 \omega^{2} \frac{\partial}{\partial \psi} \mathcal{N} \psi \frac{\partial \phi}{\partial \psi}-\frac{\omega^{2} \mathcal{N}}{2 \psi} \phi \\
-\frac{\phi \overline{\mathcal{N} \Gamma^{2}}}{\bar{\psi}}-\frac{i \omega \nu}{\bar{\psi}} \mathcal{N}_{e} \phi=0 .
\end{gathered}
$$

With $\bar{\psi}$ a constant ( $\bar{\psi}$ signifies the $\psi$ scale of the plasma),

$$
\begin{aligned}
\mathcal{N} & =\sum_{j} \int \frac{d s n_{j} m_{j}}{B^{2}} \\
\overline{\mathcal{N} A} & =\sum_{j} \int \frac{d s n_{j} m_{j} A_{j}}{B^{2}}
\end{aligned}
$$




$$
\begin{aligned}
\overline{\mathcal{N} \Gamma^{2}} & =\bar{\psi} \int \frac{d s \kappa}{B^{2} r} \frac{D\left(P_{\perp}+P_{\|}\right)}{D \psi} \\
\mathcal{N}_{e} & =\frac{m_{i} L n_{e}(L / 2)}{B_{0}^{2}(L / 2)} \\
\nu & =\frac{e^{2} \bar{\psi} v_{i} \epsilon(\psi) B_{0}(L / 2)}{\sqrt{\pi} c^{2} T_{e} m_{i} L}
\end{aligned}
$$

and we have assumed symmetry of all parameters about the midplane with limiters at $s= \pm L / 2$.

For $\psi<\psi_{0}, \epsilon(\psi)=0$, and to lowest order only the FLR term is present in Eq. (5). The leading order solution, that is regular at the origin, is $\phi=\psi^{1 / 2}$. This solution is valid over most of the plasma, but perhaps not near $\psi \simeq \psi_{0}$, where $\overline{\mathcal{N}} \bar{A}$ is becoming small. Now, without approximation at this point, let us integrate Eq. (5) in the interval $0 \leq \psi<\psi_{0}$, and with $\phi=\psi^{1 / 2}+\phi_{1}$, we find

$$
\begin{aligned}
\overline{\mathcal{N} A} \psi^{2} & \frac{\partial}{\partial \psi}\left(\frac{\phi_{1}}{\psi^{1 / 2}}\right)=-\omega^{2} \int_{0}^{\psi} d \psi^{\prime} \psi^{1 / 2} \frac{\partial}{\partial \psi} \psi^{\prime} \mathcal{N}\left(\psi^{\prime}\right) \frac{\partial}{\partial \psi^{\prime}}\left(\psi^{1 / 2}+\phi_{1}\right) \\
& +\frac{\omega^{2}}{4} \int_{0}^{\psi} d \psi^{\prime} \mathcal{N}\left(\psi^{\prime}\right)\left(1+\phi_{1} / \psi^{1 / 2}\right)+\frac{1}{2} \int_{0}^{\psi} d \psi^{\prime} \psi^{\prime} \overline{\mathcal{N} \Gamma^{2}}\left(1+\phi_{1} / \psi^{\prime 1 / 2}\right) / \bar{\psi} .
\end{aligned}
$$

We have used that $\phi_{1}(0)=0$ as required for the regularity of the solution. Further note that the right-hand side is insensitive to $\phi_{1} / \psi^{1 / 2}$ if this quantity only competes with unity near the edge of the plasma. One can infer a-posteriori that this is indeed the case if $\mathcal{N} A$ is small near the edge. Thus in Eq. (6) we an neglect $\phi_{1}$ on the right-hand side, and integrate Eq. (6) to determine $\phi_{1}\left(\psi_{0}\right)$. We find

$$
\begin{aligned}
& \left.\overline{\mathcal{N} A} \psi^{2} \frac{\partial}{\partial \psi}\left(\frac{\phi_{1}}{\psi^{1 / 2}}\right)\right|_{\psi=\psi_{0}}=-\frac{1}{2} \int_{0}^{\psi_{0}} \psi d \psi\left[\omega^{2} \frac{\partial \mathcal{N}(\psi)}{\partial \psi}-\frac{\overline{\mathcal{N} \Gamma^{2}}}{\bar{\psi}}\right] \\
& \frac{\phi_{1}\left(\psi_{0}\right)}{\psi_{0}^{1 / 2}} \doteq \frac{1}{2} \int_{0}^{\psi_{0}} \frac{d \psi}{\overline{\mathcal{N} A} \psi^{2}} \int_{0}^{\psi} d \psi^{\prime} \psi^{\prime}\left[\frac{\mathcal{N} \Gamma^{2}}{\bar{\psi}}-\omega^{2} \frac{\partial \mathcal{N}\left(\psi^{\prime}\right)}{\partial \psi^{\prime}}\right] .
\end{aligned}
$$

In the scrape-off region we assume that the FLR term balances the loss term. However, we also assume that the density drop-off in the loss region is rapid, and hence the eigenfunction 
$\phi=\psi^{1 / 2}+\phi_{1}$, does not change in the thin layer where the density is significant. Thereby, keeping only the first and last terms of Eq. (5), and integrating from $\psi=\psi_{0}$ to arbitrarily large $\psi$, we obtain assuming $\phi_{1}$ vanishes at large $\psi$

$$
\left.\overline{\mathcal{N} A} \psi_{0}^{2} \frac{\partial}{\partial \psi}\left(\frac{\phi_{1}}{\psi}\right)\right|_{\psi=\psi_{0}}=i \frac{\omega \psi_{0}}{2 \bar{\psi}}\left(1+\frac{\phi_{1}}{\psi^{1 / 2}}\right) \nu \Delta \psi \mathcal{N}_{\mathrm{e}}\left(\psi_{0}\right)
$$

with $\nu \Delta \psi \mathcal{N}_{e}\left(\psi_{0}\right)=\int_{\psi_{0}}^{\infty} d \psi \nu \mathcal{N}_{e}$. Now combining Eqs. (7)-(9) produces the dispersion relation

$$
\int_{0}^{\psi_{0}} d \psi \psi\left[\frac{\overline{\mathcal{N} \Gamma^{2}}}{\bar{\psi}}-\omega^{2} \frac{\partial \mathcal{N}}{\partial \psi}\right]+\frac{i \omega}{2} \frac{\psi_{0}}{\bar{\psi}}\left[2+\int_{0}^{\psi_{0}} \frac{d \psi}{\overline{\mathcal{N A}} \psi^{2}} \int_{0}^{\psi} d \psi^{\prime} \psi^{\prime}\left(\frac{\overline{\mathcal{N} \Gamma^{2}}}{\bar{\psi}}-\omega^{2} \frac{\partial \mathcal{N}}{\partial \psi^{\prime}}\right)\right] \nu \Delta \psi \mathcal{N}_{e}=0 .
$$

To evaluate this dispersion explicitly, we model the parameters as follows. For $\psi<\psi_{0}$ let

$$
\begin{aligned}
\mathcal{N} & =\mathcal{N}_{0} \exp \left(-\frac{\psi}{\bar{\psi}}\right), \quad \mathcal{N}_{e}=\mathcal{N}_{0} \exp \left(-\frac{\psi}{\bar{\psi}}\right), \\
\overline{\mathcal{N} \Gamma^{2}} & =\mathcal{N}_{0} \exp \left(-\frac{\psi}{\bar{\psi}}\right) \bar{\Gamma}^{2} \\
\overline{\mathcal{N} A} & =\left[-\delta \omega_{h}^{*} \omega-\omega_{h}^{*^{2}} \delta \hat{\beta} \exp \left(-\frac{\psi}{\bar{\psi}}\right) / 2\right] \mathcal{N}_{0} \exp \left(-\frac{\psi}{\bar{\psi}}\right),
\end{aligned}
$$

where $\widehat{\beta}_{h}, \mathcal{N}_{0}, \delta, \omega_{h}^{*}, \bar{\Gamma}^{2}, \nu \Delta \psi$, are constants with their significane clear from previously defined notation, and where we have emphasized the FLR dependence of the hot species and suppressed the FLR dependence of the background species. We can then integrate Eq. (10), using that $\mathcal{N}\left(\psi_{0}\right) / \mathcal{N}(0) \ll 1$, and find

$$
\bar{\Gamma}^{2}+\omega^{2}+2 i \omega \nu_{*}\left[a_{0}-\frac{\left(\bar{\Gamma}^{2}+\omega^{2}\right) / 2}{\delta_{1} \omega_{h}^{*} \omega+\delta_{2} \widehat{\beta}_{h} \omega_{h}^{*^{2}}} \frac{\exp \left(-\psi_{0} / \bar{\psi}\right) / 2}{\exp }\right]=0
$$

where $\nu^{*}=\nu \Delta \psi / 2 \psi_{0}, a_{0}=\psi_{0}^{2} \exp \left(-\psi_{0} / \bar{\psi}\right) / \bar{\psi}^{2}$ and

$$
\begin{aligned}
& \frac{1}{\delta_{1} \omega_{h}^{*} \omega+\delta_{2} \widehat{\beta}_{h} \omega_{h}^{*^{2}} \exp \left(-\psi_{0} / \bar{\psi}\right) / 2}=-\mathcal{N}_{0} \exp \left(-\frac{\psi_{0}}{\bar{\psi}}\right) \frac{\psi_{0}^{2}}{\bar{\psi}^{2}} \int_{0}^{\psi_{0}} \frac{d \psi}{\overline{\mathcal{N} A} \psi^{2}} \int_{0}^{\psi} d \psi^{\prime} \frac{\psi^{\prime}}{\bar{\psi}} \exp \left(-\frac{\psi^{\prime}}{\bar{\psi}}\right) \\
& \doteq \int_{0}^{\infty} \frac{d x \exp (-x)}{\delta \omega_{h}^{*} \omega+\delta \widehat{\beta}_{h} \omega_{h}^{*^{2}} \exp \left(-\frac{\psi_{0}}{\psi}\right) \operatorname{erp}(x) / 2} \text {. }
\end{aligned}
$$


Note that if $\omega_{h}^{*} \omega \gg \omega_{h}^{*^{2}} \hat{\beta}_{h} \exp \left(-\frac{\psi_{0}}{\bar{\psi}}\right)$, then $\delta_{1}=\delta$ and if $\omega_{h}^{*^{2}} \widehat{\beta}_{h} \exp \left(-\frac{\psi_{0}}{\bar{\psi}}\right) \gg \omega_{h}^{*} \omega$, then $\delta_{2}=\delta / 2$. Hence, a simple inter polation formula is obtained by choosing $\delta_{1}=\delta$ and $\delta_{2}=\delta / 2$. We can then rewrite our dispersion relation as

$$
\left(\delta \omega_{h}^{*} \omega+\delta \beta_{0} \omega_{h}^{*^{2}} / 2\right) \cdot\left(\omega^{2}+\bar{\Gamma}^{2}\right)-i \nu * \omega\left[\omega^{2}-2 a_{0} \delta \omega_{h}^{*} \omega+\Gamma^{2}\right]=0
$$

where $\beta_{0}=\hat{\beta}_{h} \exp \left(-\dot{\psi}_{0} / \bar{\psi}\right) / 2, \Gamma^{\prime 2}=\bar{\Gamma}^{2}-a_{0} \beta_{0} \delta \omega_{h}^{*^{2}}$. Note that $2 \beta_{0}$ is the beta value at the edge of the species with the largest finite beta FLR contribution even when our profiles are not the special choices we have taken. In particular, the $\beta_{0}$ term can be competitive with $\bar{\Gamma}^{2}$ term if a reasonable fraction of the hot particle pressure still persists near the limiter.

In the limit $\nu_{*} \rightarrow \infty$ (dominating effect from end loss), we have the solution

$$
\omega_{1,2}=a_{0} \delta \omega_{h}^{*} \pm\left[\left(a_{0} \delta \omega_{h}^{*}\right)^{2}-\Gamma^{\prime 2}\right]^{1 / 2}
$$

Thus stability is achieved if

$$
\Gamma^{2}<\left(a_{0} \delta \omega_{h}^{*}\right)^{2}
$$

However, at finite $\nu_{*}$ we find that instability persists. For example, if $\nu_{*}$ is large but finite, the frequency is $\omega_{1,2} \approx \omega_{1,2}^{(0)}+i \operatorname{Im} \omega_{1,2}$, with $\omega_{1,2}^{(0)}$ given by Eq. (13) (thi: upper sign is associated with the 1-inode) and $\operatorname{Im} \omega_{1,2}$ given by

$$
\operatorname{Im} \omega_{1,2}=\mp \frac{\left(\delta \omega_{h}^{*}+\delta \beta_{0} \omega_{h}^{*^{2}} / 2\right)^{2} \omega_{1,2}^{(0)} a_{0}}{\nu_{*}\left[\left(a_{0} \delta \omega_{h}^{*}\right)^{2}-\Gamma^{\prime 2}\right]^{1 / 2}}
$$

Note that if $\Gamma^{\prime 2}>0$, then $\omega_{1}^{(0)}$ and $\omega_{2}^{(0)}$ are the same sign and negative as $\delta \omega_{*}<0$. In this case the $\omega_{1}$ mode is unstable. If $\Gamma^{2}<0$, then the two modes are of opposite sign and with $\omega_{1}^{(0)}>0$ and $\omega_{2}^{(0)}<0$, and the modes with a finite frequency as $\nu_{*} \rightarrow \infty$ are stable. However, if $\Gamma^{\prime 2}<0$ an additional unstable root still persists, with $\omega \rightarrow 0$ as $\nu_{*} \rightarrow \infty$, we find

$$
\omega \approx i \bar{\Gamma}^{2} \beta_{0} \delta \omega_{h}^{*^{2}} / 2 \Gamma^{\prime 2} \nu_{*}
$$


In this limit the frequency is purely imaginary, and unstable when $\Gamma^{2}>0$ and $\Gamma^{2}<0$. Thus at finite $\nu_{*}$, instability still persists if $\Gamma^{2}>0$, even if $\Gamma^{2}<0$.

In Fig. 2 we present a plot of $\operatorname{Im} \omega$ vs. $\nu_{*}$ for various values of $\beta_{0}$ with $\delta \omega_{h}^{*} / \Gamma=4$, with $\omega_{h}^{*} / \Gamma=100$. Note that the low frequency unstable mode when $\Gamma^{2}<0$ appears to continuously link to the unstable modes when $\Gamma^{2}>0$.

For our specific example we assumed $\delta \gg \omega_{h}^{*} / \omega_{i}^{*}$. Frequently we can also have $\omega_{i}^{*^{2}} / \omega_{h}^{*^{2}} \ll$ $\delta \ll \omega_{i}^{*} / \omega_{h}^{*}$. In this case Eq. (12) is altered by having $\delta \omega_{h}^{*} \rightarrow \omega_{i}^{*}$ but $\delta \beta_{0} \omega_{h}^{*^{2}}$ and $\Gamma^{\prime 2}$ remaining unchanged with the rest of the analysis unaltered.

In summary, we have analyzed the $m=1$ stability of a plasma with strong FLR effects, where the edge is terminated by a limiter that causes scrape-off losses of the background plasma. These losses cause the electric fields at the edge to short, and prevent a pure displacement mode from arising, hence allowing for some FLR stabilization. However, the electric field shorting is incomplete, and instability persists if the system is unstable to ideal MHD theory. As the growth rate decreases appreciably with the limiter end loss the system should be more amenable to feedback stabilization, compared to a system without the limiter end-loss.

\section{Acknowledgment}

One of us (HLB) would like to thank Academician D.D. Ryutov for his hospitality and important scientific comments while visiting the Budker Institute of Nuclear Physics. We are also grateful to Dr. A.A. Ivanov for important conversations concerning the applicability of the theory we have presented to the Gas Dynamic Trap Experiment. This work was supported by the U.S. Department of Energy contract No. DE-FG05-80-ET-53088. 


\section{References}

${ }^{1}$ M.N. Rosenbluth, N.A. Krall, and N. Rostoker, Nuclear Fusion Suppl. Pt. 1, pp. 143-150 (1962).

${ }^{2}$ L.D. Pearlstein and N.A. Krall, Phys. Fluids 9, 2231 (i966).

3H.L. Berk, M.N. Rosenbluth, H.V. Wong, and T.M. Antonsen, Jr., Phys. Fluids 27, 2705 (1984).

4T.B. Kaiser and L.D. Pearlstein, Phys. Fluids 28, 1003 (1985).

${ }^{5}$ H.L. Berk, K.T. Tsang, H.V. Wong, Phys. Fluids 30, 2681 (1987).

${ }^{\circ}$ H.H. Flesichmann, K. Kupter, and R.E. Kreibel, Phys. Fluids 28, 1917 (1985).

${ }^{7}$ B.I. C'ohen, R.P. Freis, and W.A. Newcomb, Phys. Fluids 29, 1558-1577 (1986).

${ }^{8}$ J. Liu, W. Horton, and J.E. Sedlak, Phys. Fluids 30, 467 (1987).

${ }^{9}$ W.K. Kunkel and J.U. Guillory, in Seventh International Conference on Phenomena in Ionized Gases, Belgrade, 1965, Vol. 8, p. 702.

${ }^{10}$ W.A. Newcomb, Annals Phys. 81, 231 (1973).

${ }^{i 1}$ J.P. Freidberg and L.D. Pearlstein, Phys. Fluids 21, 1207 (1978).

${ }^{12}$ H.L. Berk and B.G. Lane, Phys. Fluids 29, 1076 (1986).

${ }^{13}$ I.A. Kotelnikov and H.L. Berk, submitted to J. Plasma Physics. 


\section{Figure Captions}

1. Schematic of open system for two-component plasma confinement. The limiters are situated outside the region occupied by hot particles. This region is coverd by dots.

2. The growth rates $\operatorname{Im} \omega$ vs $\nu_{*}$ for $\delta \omega_{*} / \Gamma=4, \omega_{* h} / \Gamma=100$ and various beta: $1, \beta_{0}=0$; $2, \beta_{0}=.01 ; 3, \beta_{0}=0.1$. 


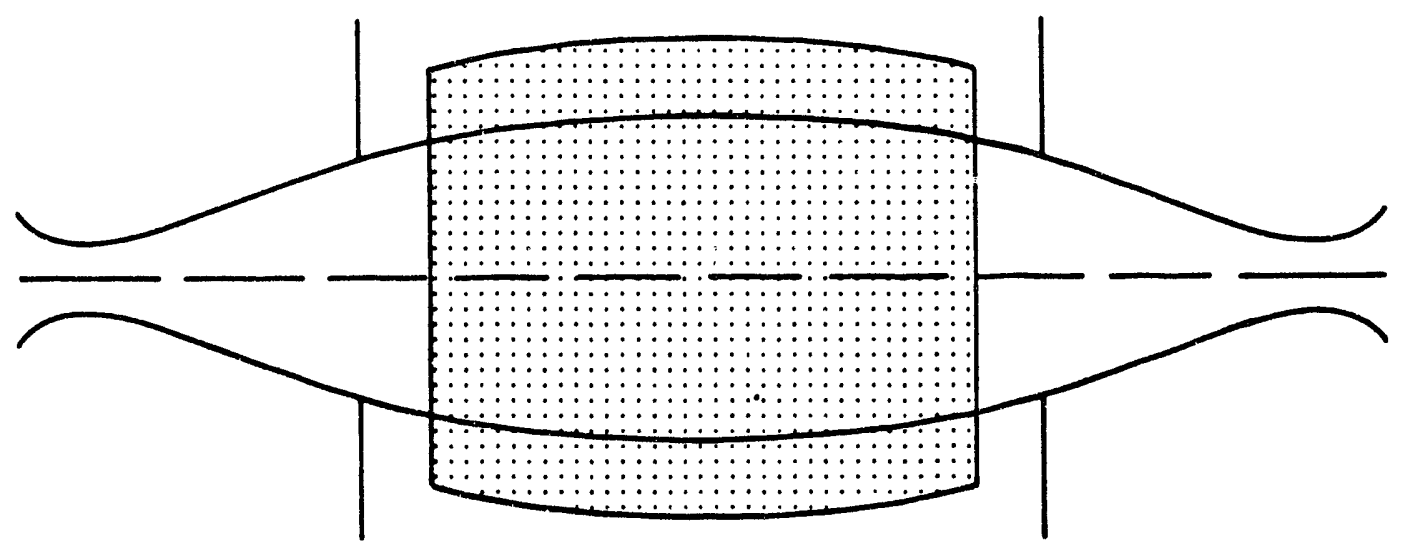




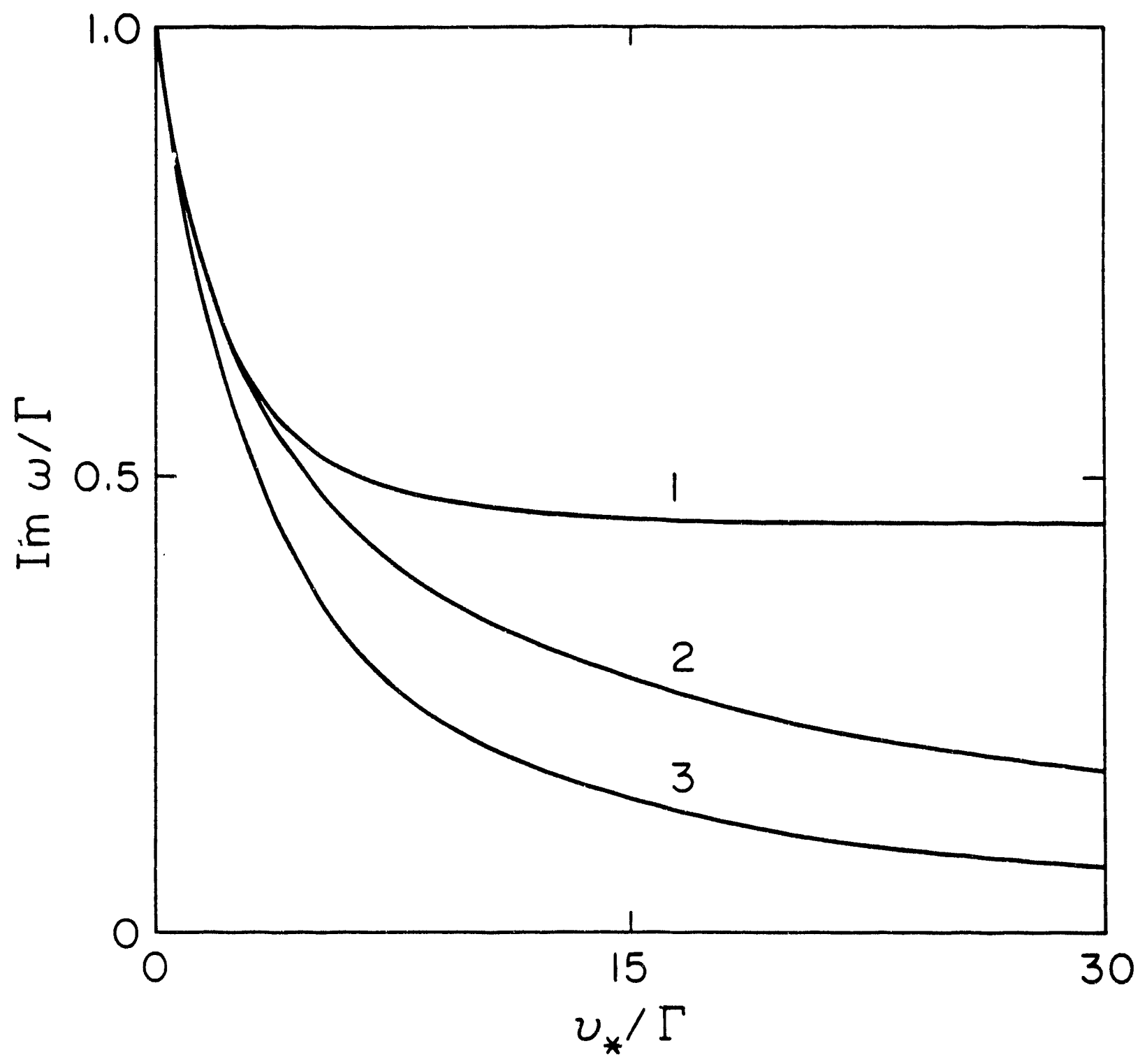



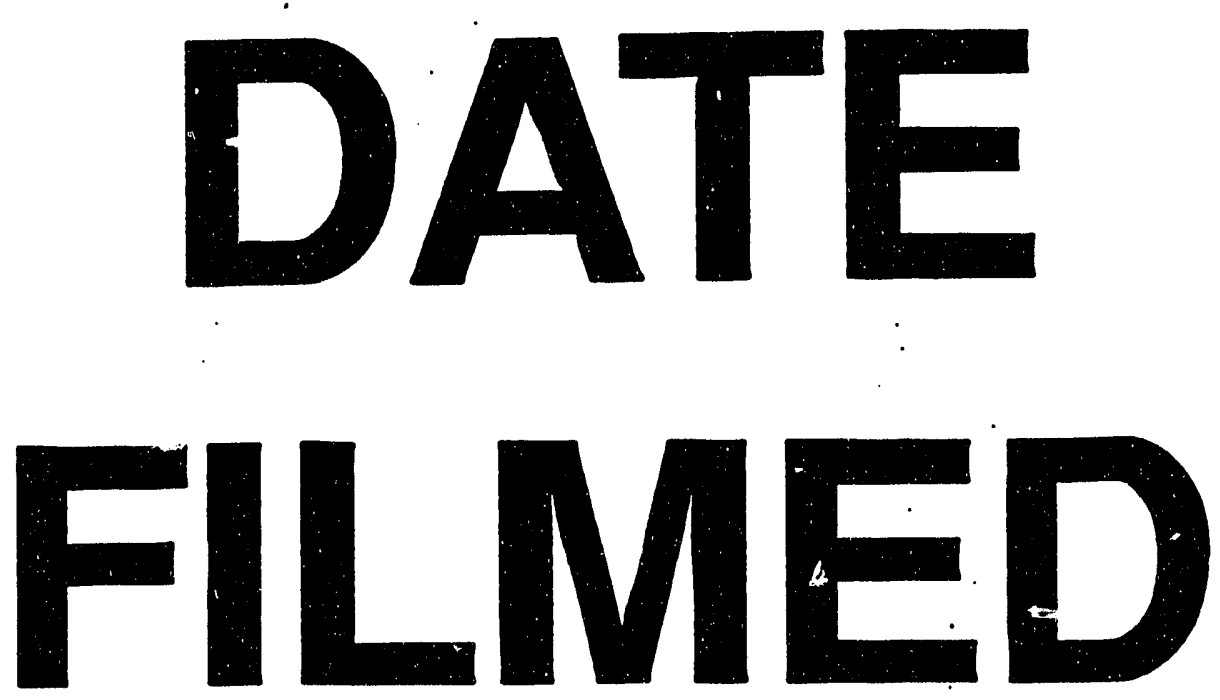

$11 / 4 / 93$
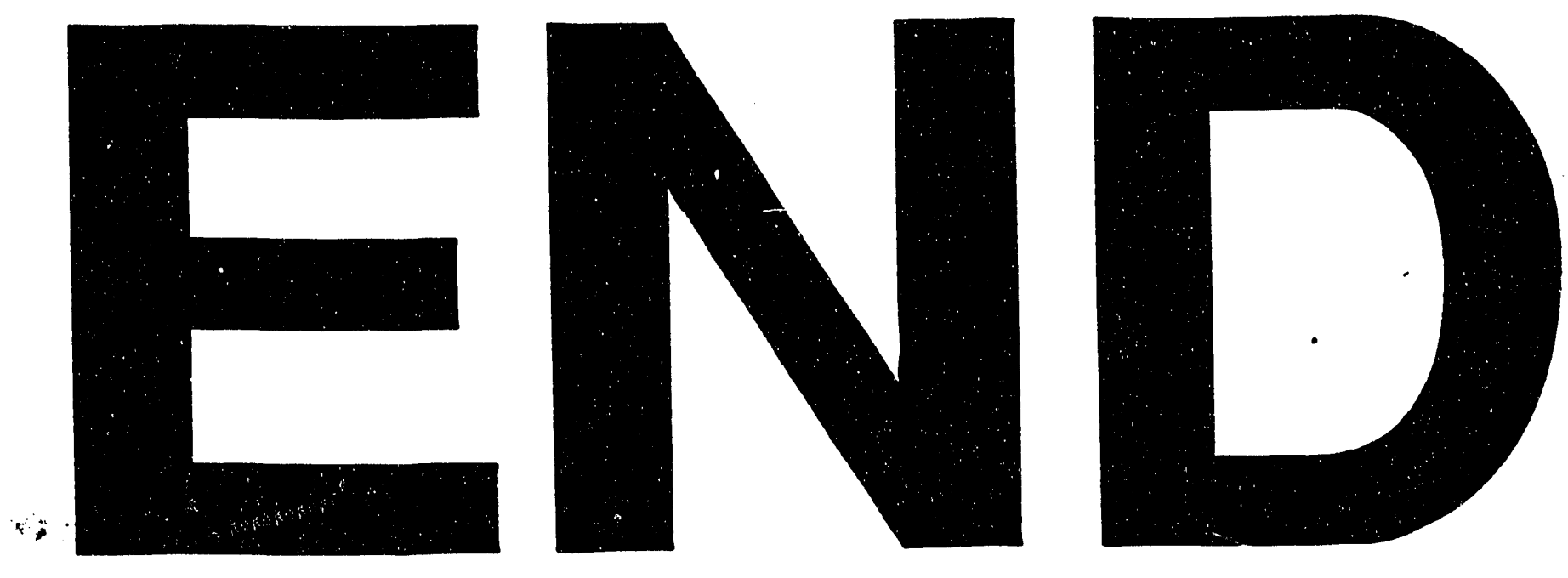
